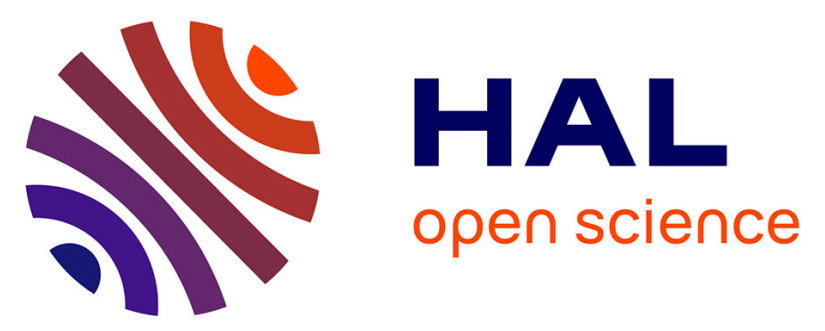

\title{
Combining label-free and label-based accurate quantifications with SWATH-MS: Comparison with SRM and PRM for the evaluation of bovine muscle type effects
}

\author{
Joanna Bons, Gauthier Husson, Marie Chion, Muriel Bonnet, Myriam \\ Maumy-Bertrand, François Delalande, Sarah Cianférani, Frédéric Bertrand, \\ Brigitte Picard, Christine Carapito
}

\section{- To cite this version:}

Joanna Bons, Gauthier Husson, Marie Chion, Muriel Bonnet, Myriam Maumy-Bertrand, et al.. Combining label-free and label-based accurate quantifications with SWATH-MS: Comparison with SRM and PRM for the evaluation of bovine muscle type effects. Proteomics, 2021, 21 (10), pp.2000214. 10.1002/pmic.202000214 . hal-03199937

\section{HAL Id: hal-03199937 https://hal.science/hal-03199937}

Submitted on 24 Nov 2021

HAL is a multi-disciplinary open access archive for the deposit and dissemination of scientific research documents, whether they are published or not. The documents may come from teaching and research institutions in France or abroad, or from public or private research centers.
L'archive ouverte pluridisciplinaire HAL, est destinée au dépôt et à la diffusion de documents scientifiques de niveau recherche, publiés ou non, émanant des établissements d'enseignement et de recherche français ou étrangers, des laboratoires publics ou privés.

\section{(c)(1)}

Distributed under a Creative Commons Attribution| 4.0 International License 


\section{Combining label-free and label-based accurate quantifications with SWATH-}

\section{MS: comparison with SRM and PRM for the evaluation of bovine muscle type}

\section{effects}

Joanna Bons ${ }^{1}$, Gauthier Husson ${ }^{1}$, Marie Chion ${ }^{1,2}$, Muriel Bonnet ${ }^{3}$, Myriam Maumy-Bertrand ${ }^{2}$, François Delalande ${ }^{1}$, Sarah Cianférani ${ }^{1}$, Frédéric Bertrand ${ }^{4}$, Brigitte Picard $^{3}$, Christine Carapito ${ }^{1}$

${ }^{1}$ Laboratoire de Spectrométrie de Masse BioOrganique (LSMBO), IPHC UMR 7178, CNRS, Université de Strasbourg, 67000 Strasbourg, France.

${ }^{2}$ Institut de Recherche Mathématique Avancée, UMR CNRS 7501, Université de Strasbourg, 67000 Strasbourg, France.

${ }^{3}$ Université Clermont Auvergne, INRA, VetAgro Sup, UMR Herbivores, 63122 Saint-Genès-Champanelle, France. ${ }^{4}$ Laboratoire de Modélisation et Sûreté des Systèmes, Institut Charles Delaunay, UMR CNRS 6281, Université de Technologie de Troyes, 12 Rue Marie Curie, 42060 Troyes, France.

Correspondence: Dr. Christine Carapito,

Laboratoire de Spectrométrie de Masse BioOrganique, IPHC UMR 7178, 25 rue Becquerel, 67087 Strasbourg, France. E-mail: ccarapito@unistra.fr. Fax: +33 368852730

Abbreviations: DDA, data-dependent acquisition; DIA, data-independent acquisition; FA, formic acid; HR/AM, high resolution/accurate mass; LLOQ; lower LOQ; LT, Longissimus thoracis muscle; QQQ, triple quadrupole; RMSE, root mean square errors; RT, retention time; SM, Semimembranosus muscle; SIL, stable isotope-labelled; ST, Semitendinosus muscle; SWATH-MS, sequential windowed acquisition of theoretical mass spectra; ULOQ, upper LOQ. 
Keywords ( $\max 5$, alphabetical order): Beef meat quality; Data-Independent Acquisition (DIA); Quantitative MS; SWATH-MS; Targeted proteomics.

Total number of words: 5966 words 


\section{Abstract}

Mass spectrometry has proven to be a valuable tool for the accurate quantification of proteins. In this study, the performances of three targeted approaches, namely Selected Reaction Monitoring (SRM), Parallel Reaction Monitoring (PRM) and Sequential Windowed Acquisition of Theoretical Mass Spectra (SWATH-MS), to accurately quantify ten potential biomarkers of beef meat tenderness or marbling in a cohort of 64 muscle samples were evaluated. Besides and so as to get the most benefit out of the complete M2 maps that are acquired in SWATH-MS, an original label-free quantification method to estimate protein amounts using an I-spline regression model was developed. Overall, SWATH-MS outperformed SRM in terms of sensitivity and dynamic range, while PRM still performed the best, and all three strategies showed similar quantification accuracies and precisions for the absolute quantification of targets of interest. This targeted picture was extended by 585 additional proteins for which amounts were estimated using the label-free approach on SWATH-MS, thus offering a more global profiling of muscle proteomes and further insights into muscle type effect on candidate biomarkers of beef meat qualities as well as muscle metabolism. 


\section{Significance of the study}

Grouped together under the terms of targeted proteomics quantification methods, SRM, PRM and SWATH-MS perform however differently in terms of data acquisition, analysis, multiplexing and performances. Notably, SWATH-MS generates comprehensive maps of the biological samples by acquiring MS2 information for all detectable precursors, while SRM and PRM targets predefined precursors. In this study, the performances of the three approaches for the absolute quantification of candidate biomarkers of beef meat tenderness or marbling were benchmarked. An original method to derive protein amount estimations for all other analytes present in the samples was also proposed, taking advantage of the exhaustive MS2 information recording in SWATH-MS mode. Hence, this demonstrates the capability of SWATH-MS to offer both label-free and label-based accurate quantifications of proteins in a single analysis. Altogether, this study also evidences that targeted proteomics is a valuable tool for finely investigating beef meat quality and bovine muscle metabolism. 


\section{Introduction}

LC-MS/MS-based quantitative proteomics has become a valuable tool to explore proteomes and gain insights into the biological systems ${ }^{[1]}$. Among the panel of available strategies, targeted approaches are preferred to accurately quantify proteins, thus enabling potential biomarker validation ${ }^{[2,3]}$ or providing fine views of system dynamics ${ }^{[4,5]}$.

Selected reaction monitoring (SRM) ${ }^{[6,7]}$, performed on triple-quadrupole (QQQ) instruments, has been considered as the gold standard for absolute quantification thanks to its high performances in terms of sensitivity, accuracy and reproducibility. With the introduction of high resolution/accurate mass (HR/AM) instruments - Q-Orbitrap and Q-TOF hybrids, additional targeted approaches have emerged, including parallel reaction monitoring (PRM) ${ }^{[8,9]}$ and sequential windowed acquisition of all theoretical fragment ion spectra-mass spectrometry (SWATH-MS) based on data-independent acquisition (DIA) ${ }^{[10]}$. SWATH-MS relies on the MS2 acquisition of all precursors across wide $\mathrm{m} / \mathrm{z}$ windows covering the entire desired $\mathrm{m} / \mathrm{z}$ range. Contrary to SRM and PRM, peptides of interest are actually targeted postacquisition during data extraction, generally however at the expense of ease of data processing ${ }^{[4,10-12]}$. In other words, as SWATH-MS is not a targeted acquisition method ${ }^{[11]}$, multiplexing capabilities are improved. This offers the possibility to obtain accurate quantification of beforehand selected proteins using stable isotope-labelled (SIL) standards ${ }^{[4,5,10,13-16]}$, as with SRM and PRM, but also to estimate proteome-wide absolute quantification in a label-free manner ${ }^{[14,17,18]}$.

Tenderness and marbling, associated with intramuscular fat content, constitute the main quality traits for beef meat conditioning consumer satisfaction and economic performances of beef production. These traits are highly variable depending on muscle type, animal (breed, gender, age) and rearing management ${ }^{[19]}$. Currently these qualities can be measured only after the slaughter of the animal by chemical quantification of intramuscular lipids (marbling), by mechanical measurements of tenderness 
or by sensory evaluation of meat perception. For several years, a strategy for biomarker discovery has been developed to identify proteins associated with tenderness or/and marbling ${ }^{[20-22]}$. The goal is to ultimately develop a prognosis tool for the evaluation and prediction of tenderness and marbling of carcasses or living animals, which could be used by the professionals of the beef sector. A list of candidate biomarkers of tenderness and/or marbling has been proposed ${ }^{[22,23]}$. In order to validate the relationships between some candidate proteins and the two quality traits on a large scale, it is necessary to be able to quantify the abundance of the proteins.

In this study, the performances of label-based SWATH-MS, SRM and PRM to accurately quantify ten candidate biomarkers of beef meat tenderness or marbling were evaluated, in a cohort of 64 bovine muscle tissues expected to cover a wide biological range of these traits. Limits of quantification, dynamic range and quantification performances were assessed. Moreover, a novel method to estimate protein amounts from SWATH-MS data in a label-free manner was introduced. The combination of this quantitative information enabled gaining insights into muscle type effect on the candidate biomarkers of beef meat qualities and muscle metabolism.

\section{Materials and methods}

\subsection{Sample preparation}

Sixty-four muscle samples from previous experiments were used ${ }^{[24-26]}$. They consist of 23 samples of Semimembranosus (SM, fast oxido-glycolytic with intermediate intramuscular fat content), 33 samples of Longissimus thoracis (LT, mixt oxidative muscle with high intramuscular fat content), and 8 samples of Semitendinosus (ST, fast glycolytic with low intramuscular fat content) muscles. These muscle samples were collected on cows and young bulls from several breeds (Rouge des Prés, Limousine, Blonde d'Aquitaine) to be representative of cattle used in the French beef production. In addition, they have been chosen to represent a wide variety of tenderness and marbling. Proteins were extracted 
and samples were prepared as described in Bonnet et al. ${ }^{[2]}$. Briefly, $30 \mu \mathrm{g}$ proteins were prepared in triplicate using a tube-gel protocol slightly adapted from Muller et al. ${ }^{[27]}$.

Eleven samples were randomly chosen as representative matrix for method development, external quality control and generating a spectral library necessary for SWATH-MS data interpretation. The matrix pool was prepared in tube-gel and fractionated by SDS-PAGE as described in Supporting Information.

For absolute quantification, a concentration-balanced mixture of 20 accurately quantified SIL peptides (Spike Tides ${ }^{\mathrm{TM}}$ TL, JPT Peptide Technologies, Berlin, Germany) was spiked in each sample. Retention time standards (iRT; Biognosys, Schlieren, Switzerland) were additionally spiked in samples analysed in DDA and SWATH-MS modes.

\subsection{Liquid chromatography and mass spectrometry}

PRM analyses were performed on a nanoAcquity UPLC device (Waters, Milford, MA) coupled to a QExactive Plus mass spectrometer (Thermo Fisher Scientific, Bremen, Germany). Five hundred ng peptides were separated on an Acquity UPLC BEH130 C18 column ( $250 \mathrm{~mm} \times 75 \mu \mathrm{m}, 1.7 \mu \mathrm{m}$ particles; Waters) at $0.45 \mu \mathrm{L} / \mathrm{min}$ coupled to a Symmetry C18 precolumn (20 mm $\times 180 \mu \mathrm{m}, 5 \mu \mathrm{m}$ diameter particles; Waters ) in a trap-elute configuration, using the following gradient of solvent B $(0.1 \%$ FA in $A C N)$ : linear from $1 \%$ to $3 \%$ in $0.5 \mathrm{~min}$, linear from $3 \%$ to $26 \%$ in $54.5 \mathrm{~min}$, linear from $26 \%$ to $35 \%$ in 5 min, and up to $90 \%$ in 1 min. A scheduled PRM method consisting of one full MS scan and 16 targeted MS2 scans was developed. The full MS scan was collected from $300-1800 \mathrm{~m} / \mathrm{z}$ at a resolution of 17,500 at $200 \mathrm{~m} / \mathrm{z}$ (AGC target: 3e6, maximum IT: $50 \mathrm{~ms}$ ). Targeted MS2 scans were collected at a resolution of 35,000 at $200 \mathrm{~m} / \mathrm{z}$ (AGC target: 1e6, maximum IT: $128 \mathrm{~ms}$ ) and scheduled for 3 min around the expected retention time (RT). 
SRM analyses were performed on a Dionex UltiMate 3000 system coupled with a TSQ Vantage (both from Thermo Fisher Scientific). Six $\mu$ g peptides were separated on a Zorbax 300SB-C18 column (150 $\mathrm{mm} \times 0.3 \mathrm{~mm}, 3.5 \mu \mathrm{m}$ diameter particles; Agilent, Santa Clara, CA) at $5 \mu \mathrm{L} / \mathrm{min}$ using the same gradient of solvent B ( $98 \% \mathrm{ACN}, 0.1 \% \mathrm{FA}$ ): linear from $5 \%$ to $25 \%$ in $47 \mathrm{~min}$, linear from $25 \%$ to $35 \%$ in $10 \mathrm{~min}$, and up to $70 \%$ in 2 min. A scheduled SRM method with 6-min time windows, 3s cycle time and 376 transitions was developed using crude stable isotope-labelled peptides (PEPotec SRM Peptides, Thermo Fisher Scientific) (Supporting Information Table S1).

DDA and SWATH-MS analyses were performed on an ekspert nanoLC 400 system coupled to a TripleTOF 6600 mass spectrometer (both from Sciex, Concord, Canada). Peptides ( $6 \mu \mathrm{g}$ for SWATH-MS analyses) were separated as previously described for SRM analyses (except solvent B is composed of $0.1 \%$ FA in ACN). A SWATH-MS method consisting of 100 variable windows (Supporting Information Table S2) covering the $200-1,600 \mathrm{~m} / \mathrm{z}$ range with an overlap of $1 \mathrm{~m} / \mathrm{z}$ was developed. MS1 spectra were collected for $150 \mathrm{~ms}$, and MS2 spectra for $45 \mathrm{~ms}$ in high-sensitivity mode.

Details about LC-MS/MS setups are given in Supporting Information.

\subsection{Data analysis}

Spectral library generation. Identifications were performed using Mascot (version 2.5.1; Matrix Science, London, UK). The spectral library (SL) was generated in Skyline ${ }^{[28]}$ (version 3.7.1.11099) by importing Mascot result files and fixing a cut-off score of 0.95 . Details are given in Supporting Information. 
Selection of 10 candidate biomarkers and proteotypic peptides. Ten candidate biomarkers of tenderness or marbling were selected according to the criteria defined in Bonnet et al. ${ }^{[2]}$. The list of the targeted proteins and peptides is reported in Supporting Information Table S3.

Label-based quantification data processing. PRM, SRM and SWATH-MS data were processed with Skyline using appropriate settings. Chromatographic peaks were investigated to manually adjust peak integration boundaries and remove interfered transitions (at least three transitions were kept for each precursor). For each method, peptides' limit of detection (LOD) and limits of quantification (LOQ) were determined using 8-points calibration curves. After ensuring that peptides are within their linear range, the ratios between endogen and SIL peptides were used to determine the mol amount of endogenous peptides. Results are reported in $\mathrm{fmol} / \mu \mathrm{g}$ of muscular protein hereafter to limit bias towards the chromatographic system. Details are given in Supporting Information.

Label-free quantification data processing. SWATH-MS data was processed with Skyline using appropriate settings. Validated proteotypic peptides were extracted using the same parameters as for label-based quantification. Details are given in Supporting Information. Peaks were reintegrated using the target decoy approach of the mProphet peak-scoring model ${ }^{[29]}$, and a q-value was assigned to each peak. Only precursors with a q-value below 0.01 were kept, and peptide intensity was obtained by summing all precursor intensities. Quantity prediction was performed using monotone spline smoothing ${ }^{[30]}$. Monotone spline smoothing combines I-spline regression analysis and non negative least squares estimation to ensure monotonicity. Parameters of the regression models were estimated using the Lawson-Hanson algorithm for non-negative least square estimation. I-spline analysis was conducted using iSpline function from the "splines2" package ${ }^{[31]}$ and non negative least squares models were fitted using the nnls function from the "nnls" package ${ }^{[32]}$ in R 3.6 .2 software. In addition panel plots were created using the "lattice" package ${ }^{[33]}$. No prediction were computed nor derived for intensity values lying outside of the observed intensity range. Mol estimations of oxidized peptides 
and their counterparts were summed, and the amount estimations of the two most abundant peptides were averaged to obtain individual protein mol estimations.

Statistical analysis. Statistical analyses were carried out using R 3.6.2 software. For method comparison, the difference between the three groups was analysed using one-way ANOVA followed by Tukey's post-hoc test using the "stats" package ${ }^{[34]}$ (significance level set at $p \leq 0.05$ ) or using Welch t-test (significance level adjusted for multiple corrections set at $p \leq 0.017$ ). For muscle effect analysis, one-way ANOVA was performed for each protein abundance assayed by quantitative PRM to evaluate their dependence on the muscle. Then, a Tukey test was performed when the result of ANOVA was significant $(p \leq 0.05)$, using the "agricolae" package ${ }^{[35]}$. The same analysis was done on the semiquantitative protein abundance assayed by SWATH-MS, when the protein was identified in at least $80 \%$ of the samples.

\section{Results and discussion}

In this study, we compared and evaluated the performances of SWATH-MS, SRM and PRM for the accurate quantification of 10 candidate biomarkers of beef meat tenderness or marbling in a cohort of various bovine muscle tissues, chosen to represent the biological variations of tenderness and marbling (Supporting Information Fig. S1). A concentration-balanced mixture of SIL peptides was set up and optimised to favour peptide response, and 20 peptides, representing the 10 proteins of interest, were selected to be synthetized as accurately quantified SIL peptides. Note that the same concentrationbalanced mixture was used for SRM, PRM and SWATH-MS analyses.

\subsection{Sensitivity and dynamic range of targeted assays}

Calibration curves were generated to determine LOD and LOQ for the 20 peptides (Supporting Information. Fig. S2-4). To note, the peptide LAPEFAK (PRDX6) was absent from the spectral library, 
and thus could not be queried in the SWATH-MS data. Hereafter are qualified as quantifiable, peptides for which calibration curves were successfully established.

Figure 1A displays the LOD of the targeted peptides in the assays. Values ranged from 0.013 to 1,250 $\mathrm{fmol} / \mu \mathrm{g}$ of muscular proteins in SRM, 0.26 to $625 \mathrm{fmol} / \mu \mathrm{g}$ in PRM and 6.25 to $6,250 \mathrm{fmol} / \mu \mathrm{g}$ in SWATHMS (Supporting Information Table S4). LOD values were the lowest in SRM for 16 out of 19 peptides confirming the unequalled sensitivity of the double isolation in QQQ instruments. However, observations differ regarding the lower LOQ (LLOQ) ranging from 12.5 to $1,250 \mathrm{fmol} / \mathrm{\mu g}$ of muscular

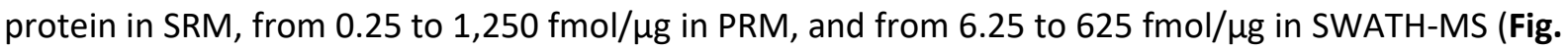
1B and Supporting Information Table S4). The lowest LLOQ were always achieved in PRM. Indeed, among the 15 common quantifiable peptides, 87\% (13) of the LLOQ were lower in PRM only, and 13\% (2) in both PRM and SWATH-MS.

Although SWATH-MS is reported in the literature to be generally less sensitive than SRM and PRM $[3,4,10,12,13]$, especially for low-abundant peptides, it appeared more sensitive than SRM in this study, with a median 3-fold increase. In line with this, Nakamura et al. observed that SWATH-MS was more sensitive than SRM ${ }^{[5]}$. PRM exhibited the best performances regarding sensitivity, both by the number of quantifiable peptides and by reaching a sensitivity down to $250 \mathrm{amol} / \mathrm{\mu g}$ of muscular protein, i.e. 125 amol injected. Gallien et al. obtained better performances of PRM regarding LLOQ than SRM as well ${ }^{[36]}$. It is worth noting that this remains peptide- and sample-dependent.

This highlights that technical improvement related to HR/AM instruments compared to QQQ instruments, both operating in a pure targeted mode, increases selectivity and hence allows better discriminating true signal from interferences. This gain in selectivity improves in fine sensitivity ${ }^{[36]}$. Moreover, one should point out the benefit of strategies relying on post-acquisition transition refinement, as PRM and SWATH-MS; improving sensitivity in SRM would contrarily require designing a new assay. 
Upper LOQ (ULOQ) values ranged from 625 to $6,250 \mathrm{fmol} / \mu \mathrm{g}$ of muscular proteins in SRM and in PRM, and 3,125 to 6,250 in SWATH-MS (Fig. 1D and Supporting Information Table S4). The dynamic ranges obtained for the quantifiable peptides covered 0.7 to 2 orders of magnitude in SRM, and 0.7 to 3.7 orders of magnitude in PRM and 1 to 2.7 orders of magnitude in SWATH-MS (Fig. 1E and Supporting Information Table S4). The widest dynamic ranges were obtained in PRM for 13 of 16 peptides, when compared to SRM and/or SWATH-MS. Concerning the 15 common quantifiable peptides, $80 \%$ (12) of the dynamic ranges were wider in PRM only, and 20\% (3) in both PRM and SWATH-MS.

This demonstrates that PRM covered wider dynamic ranges than SRM and SWATH-MS, and that PRM and SWATH-MS outperformed SRM performances. This is explained by the sensitivity performances of the three strategies, since the same ULOQ - except for QAFQIGSPWR (ALDH1A1) - were reached in PRM, SRM and SWATH-MS (Fig. 1D).

\subsection{Quantification linearity and accuracy}

Using the accurately quantified SIL peptides, peptide mol amounts were determined in the samples if (i) the peptide is quantifiable and (ii) the determined amount falls within the peptide linear range. Out of the 20 targeted peptides, 16 were finally quantified in PRM, 11 in SRM, and 13 in SWATH-MS, in at least one sample of the whole cohort, that is in line with the sensitivity performances discussed above (Supporting Information Table S5). Indeed, all peptides from ALDH1A1, QAFQIGSPWR and LECGGGPWGNK, were below the LLOQ in the whole cohort, as determined by PRM, SRM and SWATHMS. Three other peptides, VIVVGNPANTNCLTASK and LGVTSDDVK from MDH1 and VVLAYEPVWAIGTGK from TPI1, were also below the LLOQ in all samples in the SRM assay, and VIISLQLTAEK from PRDX6 in the SWATH-MS one. Besides this, 2, 10 and 6 additional peptides were below the LLOQ in PRM, SRM and SWATH-MS respectively, and 3 others were above the ULOQ in PRM, in some samples. To note, 3 and 1 peptide(s) presented some interfered signals in PRM and SWATHMS respectively, and corresponding values were excluded from the quantification assay. 
The comparisons of the quantification values are displayed in Fig. 2A-C. Only values measured by all three methods were used, representing a total of 329 values. The comparison of SRM and SWATH-MS showed very high correlation $\left(R^{2}=0.99\right)$. SRM and PRM on the one hand, and SWATH-MS and PRM on the other hand appeared to be less correlated $\left(R^{2}=0.83\right.$ for both when the peptide NNLGELINTLNAAK (TPI1) is excluded from the assay (Fig. 2A-B); $R^{2}=0.75$ and 0.74 resp. while considering it (Supporting Information Figure S5)).

Then, the ratios of quantification values were calculated $(\mathrm{N}=339$ for SRM/PRM, 720 for SWATHMS/PRM and 344 for SWATH-MS/SRM) (Fig. 2D-F). They ranged from 0.27 to 5.21 for SRM/PRM, from 0.21 to 5.82 for SWATH-MS/PRM, and from 0.84 to 1.30 for SWATH-MS/SRM. Medians equalled 0.89, 0.88 and 1.01, and means 1.20, 1.05 and 1.01 for SRM/PRM, SWATH-MS/PRM and SWATH-MS/SRM respectively. SRM/PRM ratios were significantly higher than SWATH-MS/SRM ratios $(p<0.005)$ and SWATH-MS/PRM ones $(p<0.005)$. But, the difference between SWATH-MS/PRM and SWATH-MS/SRM ratios was not significant $(p \sim 0.74)$.

Overall, 83\% (282) ratios were accurate within a factor 2.5 between SRM and PRM, and 89\% (638) between SWATH-MS and PRM, highlighting a good quantification accuracy of the three methods. It is worth noting that all values above 2.5 were obtained for the peptide YEINVLYNR (TNNT1) (values ranging between 1.69-5.21 for SRM/PRM and 1.45-5.82 for SWATH-MS/PRM more specifically), even though very high ratio dot-p values were obtained in the three different assays (rdot- $p \geq 0.97$ ). Values below 0.4 were shared between two peptides in SRM and four peptides - among which the two found in SRM - in SWATH-MS. All ratio values were accurate within a factor 1.3 between SWATH-MS and SRM, representing an excellent quantification accuracy of SWATH-MS and SRM in line with the linearity performances achieved with both methods.

PRM showed a trend to over-estimate peptide amounts, evidenced by the $25^{\text {th }}$ and $75^{\text {th }}$ percentiles equalling 0.71 and 1.05 for SRM/PRM, and 0.68 and 1.05 for SWATH-MS/PRM. This can be justified by the various instrument geometries used here. Indeed, contrary to QQQ (SRM) and Q-TOF (SWATH-MS) 
instruments based on a linear configuration, Q-Orbitrap (PRM) instruments are equipped with a trapping device, the HCD cell, in which fragment ions' are accumulated and stored, before being transferred to the C-trap with limited capacity, and eventually injected into the Orbitrap.

\subsection{Precision and reproducibility of the strategies and platforms}

A quality control sample corresponding to the spike-in of the concentration-balanced mixture of SIL peptides in a matrix representative of the cohort was daily injected onto the different couplings. Boxplots of the coefficients of variation (CV) of the light/heavy peptide area ratios are displayed in Fig. 3A. No significant differences were observed when comparing CVs obtained in PRM, SRM and SWATHMS (as determined by one-way ANOVA analysis (Fig. 3A) and by Welch t-test (Supporting Information Fig. S6)). All values were below 20\%, except for AQELSDWIHQLESEK (TNNT1) in PRM and SWATH-MS (42.6\% and $28.4 \%$ resp.) and LECGGGPWGNK (ALDH1A1) in SRM (25.7\%), which is explained by peptides' detection issue. The $25^{\text {th }}$ and $75^{\text {th }}$ percentiles equalled $1.86 \%$ and $3.22 \%$ in PRM, $2.72 \%$ and 4.89\% in SRM, and $5.34 \%$ and $12.44 \%$ in SWATH-MS, showing a higher dispersion of CV values with the latter method. This was mostly due to the Q-TOF mass spectrometer soiling. The fact that the same LC conditions were used in SRM shows that QQQ instruments are more robust for large cohort analysis.

RT stability of the different couplings are reported in Fig. 3B. RT shift represented less than 2.6 min for all peptides, regardless of the method used. The boxplots of CV of peptide RTs showed medians of $0.64 \%$ in PRM, $0.96 \%$ in SRM and $0.56 \%$ in SWATH-MS, with a maximal value of $3.0 \%$. This demonstrates the excellent RT reproducibility achievable in targeted MS analyses, a crucial parameter for time-scheduled acquisition strategies. 


\subsection{Added value of SWATH-MS for accurate label-free quantification}

In order to take advantage of the exhaustive nature of the SWATH-MS acquisition mode, quantification values were estimated for all proteins present in the samples, and not only for the 10 proteins of interest. In this regard, Schubert et al. ${ }^{[17]}$ and He et al. ${ }^{[18]}$ proposed strategies to estimate proteomewide accurate quantification on SWATH-MS/DIA, namely by assuming a linear correlation between summed MS2 intensities and accurate concentrations of anchor proteins, and by relying on the TPA algorithm ${ }^{[37,38]}$. Here, an I-spline regression model was fitted for each sample using data from labelbased quantification. The log-transformed peptide quantity was chosen as the dependant variable and the log-transformed peptide intensity as the predictor (Fig. $\mathbf{4}$ and Supporting Information Fig. S7 and Table 56). As expected, strong non-linear relation between both variables was observed and all fitted models were increasing. The monotone spline models outperform the linear regression models in terms of root mean square errors (RMSE) for 63 out of the 64 considered samples (Supporting Information Fig. S8). An exact binomial test was performed: with a 95\% confidence level, there is a probability of at least $93 \%$ that monotone spline provides a lower RMSE than linear regression. A Top2 strategy was finally applied on predicted peptide amounts to estimate protein amounts. The accuracy between the label-based and label-free accurate quantifications was assessed on the candidate biomarkers: $53 \%$ of the amount estimations were consistent within a factor 2 with the absolute labelbased quantification (Supporting Information Fig. S9). More particularly, high consistency $\left(R^{2} \geq 0.70\right)$ between both approaches was obtained for $33 \%$ of the samples, and even $83 \%$ of them while excluding the previously highlighted TNNT1 (Supporting Information Fig. S10-11). To note, the label-free approach uses the Top2 peptides, but not necessarily the label-based one, which can explain the small discrepancy between the two quantification strategies. Amounts were estimated for 585 additional proteins (296 proteins per sample in average), and ranged between 6.36 and 2,074 fmol/ $\mu \mathrm{g}$ (Supporting Information Fig. S12 and Table S7). Hence, the established accurate label-free 
quantification strategy offers a global profiling of the bovine muscle proteomes, and thus allows gaining insights into muscle metabolism.

\subsection{Muscle type effect of candidate biomarkers of beef tenderness or marbling}

The analysis of muscle type (SM, LT, ST) effect on the abundance of the proteins was performed on a subset of 51 samples including only Rouge des Prés cows to overcome the effects of animal type and rearing practices

A first differential analysis was conducted using exclusively absolute quantification values obtained on the 10 proteins of interest thanks to the PRM assay (Table 1). The most important differences were observed between LT muscle (a slow oxidative muscle) and the two others: SM (mixt oxido-glycolytic) and ST (fast glycolytic). Protein FHL1 (Four and a half LIM domains protein 1) was more abundant in LT than in SM and ST. As FHL1 has been described to be positively related to intramuscular fat content

${ }^{[22]}$, the highest abundance observed in the LT muscle with higher lipids content than the two other muscles, is logical. HSPB1 (HSP27, from small HSP family) was significantly less abundant in SM muscle. ENO3 (Enolase 3, glycolytic enzyme) was less abundant (only for peptide 2) in LT and more abundant in SM, the ST being intermediate. As the LT muscle from Rouge des Prés breed contains few or nor type IIX fibres (fast glycolytic) ${ }^{[19]}$, it is logical to observe the lowest abundance of ENO3 in this muscle. In coherence with that, TPI1 (Triose phosphate isomerase, glycolytic enzyme) and MyH1 (Myosin heavy chain IIx expressed in fast glycolytic fibres) were the most abundant in ST muscle, while MDH1 (oxidative enzyme) was less abundant in this glycolytic muscle. These results are coherent with the contractile properties of these three muscles and are in accordance with the results of Picard et al. ${ }^{[25]}$ obtained by a semi-quantitative method of Reverse Phase Protein Array on cows from the same breeds. Moreover, although the differences among muscles did not reach significance for three proteins: CRYAB (alphaBcrystalin from small HSP family), PRDX6 (peroxiredoxin 6 anti-oxidant enzyme) and TNNT1 (Troponin T slow), the ranking of their abundance among muscles is consistent with Picard 
et al. ${ }^{[25]}$. Indeed, PRDX6 was less abundant in LT than in SM and ST; TNNT1 was less abundant in ST in accordance with the low abundance of slow fibres in this muscle and CRYAB was more abundant in LT muscle in accordance with the higher proportion of slow fibres expressing CRYAB in LT muscle.

To extent the differential analysis, the quantity estimates extracted for all proteins detected in the SWATH-MS assay were considered. This analysis revealed that the abundance of proteins related to glycolytic and oxidative pathways according to the three muscles were consistent with the metabolic and contractile properties of the LT, SM and ST muscles (Table 1). Indeed, of the 585 proteins quantified in the three muscles, six (GAPDHS, GAPDH, ENO1, PKM, GPI, PGK1) were annotated by the related GO term, GO:0006096 glycolytic process. Of these, phosphoglycerate kinase 1 (PGK1), pyruvate kinase (PKM), and glucose-6-phosphate isomerase (GPI), were quantified in more than $80 \%$ of the 51 muscles, and as expected were less abundant in less glycolytic muscle LT and higher abundant in the glycolytic ST and SM muscles. The low abundance of PKM, GPI and PGK1 in the oxidative high marbled LT muscle was also consistent with the negative correlation between the abundance of these proteins and the IMF values reported by Bazile et al. ${ }^{[22]}$. Among proteins annotated by the GO term (GO:0006099) involved in tricarboxylic acid cycle, FH, DLST and MDH2 were less abundant in the ST muscle, LT and SM being equal in accordance with the contractile and metabolic properties of these muscles described in the literature ${ }^{[39]}$.

Higher abundances of proteins involved in additional oxidative metabolisms such as fatty acid betaoxidation (GO:0006635), mitochondrial electron transport, ubiquinol to cytochrome c (complex 2 of the respiratory chain, GO:0006122) and mitochondrial electron transport, cytochrome c to oxygen (complex 3 of the respiratory chain, GO:0006123) were quantified in LT than in ST or SM muscles, which is in accordance with the oxidative properties of this muscle.

Consistently, the contractile protein TNNT3 (fast isoform) was, as expected, significantly higher in ST containing higher proportion of fast glycolytic fibres, and lower in LT muscle containing few or no fast glycolytic fibres, the SM being intermediate ${ }^{[39]}$. 
In summary, the differences observed between the three muscles for the 11 proteins mentioned in Table 1 are all consistent with the contractile and metabolic properties of the muscles: the ST contained the fewest proteins associated with the slow oxidative type and the most proteins linked to the rapid glycolytic type, the opposite is observed in the $L T$, the SM being intermediate.

\section{Concluding remarks}

Only a handful of comparisons of three targeted proteomics methods can be found in the literature ${ }^{[5,40,41]}$. In this study, we have conducted a comparison of SRM, PRM and SWATH-MS assays developed and applied on a sample cohort of bovine muscle tissues. The first intention was to evaluate the performances of the three different assays for the accurate quantification of ten candidate biomarkers of beef meat tenderness or marbling using stable isotope-labelled standards.

Targeted assays were performed on different couplings (microLC-TSQ Vantage for SRM, nanoLC-QExactive Plus for PRM, and microLC-TripleTOF 6600 for SWATH-MS). By normalising fmol amount by the quantity of injected material and performing data analysis with Skyline, we assumed to have reasonably limited bias toward instrumentation to perform a fair comparison of the three quantification methods. Nevertheless, the chromatographic scale (nano- vs. micro-) has inevitably influenced quantification performances, as exemplified for few peptides. Under the conditions of our study, PRM provided the best sensitivity and dynamic range performances, and SWATH-MS outperformed SRM regarding these metrics. However, all three strategies showed quite similar quantification precision and accuracy performances, although the linear correlation between SRM and SWATH-MS quantification was higher in this context.

Then, we extended the proteome coverage by adding extracted protein amount estimates for all proteins detected in SWATH-MS using a universally applicable approach. This offered a proteome-wide quantitative repertoire of the samples. Of course, profiling comprehensiveness in SWATH-MS depends 
on the SL used for data extraction. Its building is certainly time-consuming, fortunately some SL are publicly available, but not all organisms are represented - Bos taurus is not to mention.

In conclusion, the valuable biological output of the study, demonstrated by the great coherence with previous data of the literature, validates both quantification approaches to further study, on a large scale, the relationships between the abundance of candidate biomarkers and some meat quality traits such as tenderness and marbling. 


\section{Associated data}

Complete DDA datasets have been deposited to the ProteomeXchange Consortium via the PRIDE ${ }^{[42]}$ partner repository with the dataset identifier PXD020638 and 10.6019/PXD020638.

The full Skyline data files and raw data are publicly available on Panorama public (https://panoramaweb.org/SWATH-MS_PRM_SRM_BovineMuscles.url, ProteomeXchange ID PXD020680). 
JB was financed by a fellowship from the French Ministry of Science. The authors thank the SICA Rouge des Prés, especially A. Valais and G. Aminot, for animal handling, and sampling. We thank N. GuivierFredot for protein extraction and Julien Soulat for his involvement in statistical analysis. This work was supported by INRAE-Phase Division (Funding of the RefQuant project) and by the French Proteomic Infrastructure (ProFI; ANR-10-INBS-08-03).

The authors have declared no conflict of interest. 


\section{References}

[1] Aebersold, R., \& Mann, M. (2016). Mass-spectrometric exploration of proteome structure and function. Nature, 537(7620), 347-355. doi: 10.1038/nature19949

[2] Bonnet, M., Soulat, J., Bons, J., Leger, S., De Koning, L., Carapito, C., \& Picard, B. (2020). Quantification of biomarkers for beef meat qualities using a combination of Parallel Reaction Monitoring- and antibody-based proteomics. Food Chemistry, 317. doi: ARTN 126376

10.1016/j.foodchem.2020.126376

[3] Wu, W., Dai, R., \& Bendixen, E. (2019). Comparing SRM and SWATH methods for quantitation of bovine muscle proteomes. J Agric Food Chem. doi: 10.1021/acs.jafc.8b05459

[4] Schmidlin, T., Garrigues, L., Lane, C. S., Mulder, T. C., van Doorn, S., Post, H., .. Altelaar, A. F. (2016). Assessment of SRM, MRM(3) , and DIA for the targeted analysis of phosphorylation dynamics in non-small cell lung cancer. Proteomics, 16(15-16), 2193-2205. doi: 10.1002/pmic.201500453

[5] Nakamura, K., Hirayama-Kurogi, M., Ito, S., Kuno, T., Yoneyama, T., Obuchi, W., . . Ohtsuki, S. (2016). Large-scale multiplex absolute protein quantification of drug-metabolizing enzymes and transporters in human intestine, liver, and kidney microsomes by SWATH-MS: Comparison with MRM/SRM and HR-MRM/PRM. Proteomics, 16(15-16), 2106-2117. doi: 10.1002/pmic.201500433

[6] Picotti, P., Rinner, O., Stallmach, R., Dautel, F., Farrah, T., Domon, B., . . Aebersold, R. (2010). Highthroughput generation of selected reaction-monitoring assays for proteins and proteomes. Nat Methods, 7(1), 43-46. doi: 10.1038/nmeth.1408

[7] Picotti, P., \& Aebersold, R. (2012). Selected reaction monitoring-based proteomics: workflows, potential, pitfalls and future directions. Nat Methods, 9(6), 555-566. doi: 10.1038/nmeth.2015 
[8] Peterson, A. C., Russell, J. D., Bailey, D. J., Westphall, M. S., \& Coon, J. J. (2012). Parallel reaction monitoring for high resolution and high mass accuracy quantitative, targeted proteomics. Mol Cell Proteomics, 11(11), 1475-1488. doi: 10.1074/mcp.0112.020131

[9] Gallien, S., Duriez, E., Crone, C., Kellmann, M., Moehring, T., \& Domon, B. (2012). Targeted proteomic quantification on quadrupole-orbitrap mass spectrometer. Mol Cell Proteomics, 11(12), 1709-1723. doi: 10.1074/mcp.0112.019802

[10] Gillet, L. C., Navarro, P., Tate, S., Rost, H., Selevsek, N., Reiter, L., ... Aebersold, R. (2012). Targeted data extraction of the MS/MS spectra generated by data-independent acquisition: a new concept for consistent and accurate proteome analysis. Mol Cell Proteomics, 11(6), 0111 016717. doi: 10.1074/mcp.0111.016717

[11] Borras, E., \& Sabido, E. (2017). What is targeted proteomics? A concise revision of targeted acquisition and targeted data analysis in mass spectrometry. Proteomics, 17(17-18). doi: 10.1002/pmic.201700180

[12] Ludwig, C., Gillet, L., Rosenberger, G., Amon, S., Collins, B. C., \& Aebersold, R. (2018). Dataindependent acquisition-based SWATH-MS for quantitative proteomics: a tutorial. Mol Syst Biol, 14(8), e8126. doi: 10.15252/msb.20178126

[13] Liu, Y., Huttenhain, R., Surinova, S., Gillet, L. C., Mouritsen, J., Brunner, R., . . Aebersold, R. (2013). Quantitative measurements of $\mathrm{N}$-linked glycoproteins in human plasma by SWATH-MS. Proteomics, 13(8), 1247-1256. doi: 10.1002/pmic.201200417

[14] Husson, G., Delangle, A., O'Hara, J., Cianferani, S., Gervais, A., Van Dorsselaer, A., . . Carapito, C. (2018). Dual Data-Independent Acquisition Approach Combining Global HCP Profiling and Absolute Quantification of Key Impurities during Bioprocess Development. Anal Chem, 90(2), 1241-1247. doi: 10.1021/acs.analchem.7b03965

[15] Litichevskiy, L., Peckner, R., Abelin, J. G., Asiedu, J. K., Creech, A. L., Davis, J. F., . . Jaffe, J. D. (2018). A Library of Phosphoproteomic and Chromatin Signatures for Characterizing Cellular 
Responses to Drug Perturbations. Cell Syst, 6(4), 424-443 e427. doi: 10.1016/j.cels.2018.03.012

[16] Bruderer, R., Muntel, J., Muller, S., Bernhardt, O. M., Gandhi, T., Cominetti, O., ... Reiter, L. (2019). Analysis of 1508 Plasma Samples by Capillary-Flow Data-Independent Acquisition Profiles Proteomics of Weight Loss and Maintenance. Mol Cell Proteomics, 18(6), 1242-1254. doi: 10.1074/mcp.RA118.001288

[17] Schubert, O. T., Ludwig, C., Kogadeeva, M., Zimmermann, M., Rosenberger, G., Gengenbacher, M., ... Aebersold, R. (2015). Absolute Proteome Composition and Dynamics during Dormancy and Resuscitation of Mycobacterium tuberculosis. Cell Host Microbe, 18(1), 96-108. doi: 10.1016/j.chom.2015.06.001

[18] He, B., Shi, J., Wang, X., Jiang, H., \& Zhu, H. J. (2019). Label-free absolute protein quantification with data-independent acquisition. J Proteomics, 200, 51-59. doi: 10.1016/j.jprot.2019.03.005

[19] Couvreur, S., Le Bec, G., Micol, D., \& Picard, B. (2019). Relationships Between Cull Beef Cow Characteristics, Finishing Practices and Meat Quality Traits of Longissimus thoracis and Rectus abdominis. Foods, 8(4). doi: 10.3390/foods8040141

[20] Picard, B., \& Gagaoua, M. (2017). Proteomic Investigations of Beef Tenderness. 177-197. doi: 10.1016/b978-0-12-804007-2.00011-4

[21] Ceciliani, F., Lecchi, C., Bazile, J., \& Bonnet, M. (2018). Proteomics Research in the Adipose Tissue. 233-254. doi: 10.1007/978-3-319-69682-9_12

[22] Bazile, J., Picard, B., Chambon, C., Valais, A., \& Bonnet, M. (2019). Pathways and biomarkers of marbling and carcass fat deposition in bovine revealed by a combination of gel-based and gelfree proteomic analyses. Meat Sci, 156, 146-155. doi: 10.1016/j.meatsci.2019.05.018

[23] Picard, B., \& Gagaoua, M. (2019). Meta-proteomics for the discovery of protein biomarkers of beef tenderness: an overview of integrated studies. Food Research International, in press.

[24] Picard, B., Gagaoua, M., Micol, D., Cassar-Malek, I., Hocquette, J. F., \& Terlouw, C. E. (2014). Inverse relationships between biomarkers and beef tenderness according to contractile and 
metabolic properties of the muscle. I Agric Food Chem, 62(40), 9808-9818. doi: $10.1021 / \mathrm{jf501528s}$

[25] Picard, B., Gagaoua, M., Al-Jammas, M., De Koning, L., Valais, A., \& Bonnet, M. (2018). Beef tenderness and intramuscular fat proteomic biomarkers: muscle type effect. PeerJ, 6, e4891. doi: $10.7717 /$ peerj.4891

[26] Picard, B., Gagaoua, M., Al Jammas, M., \& Bonnet, M. (2019). Beef tenderness and intramuscular fat proteomic biomarkers: Effect of gender and rearing practices. J Proteomics, 200, 1-10. doi: 10.1016/j.jprot.2019.03.010

[27] Muller, L., Fornecker, L., Van Dorsselaer, A., Cianferani, S., \& Carapito, C. (2016). Benchmarking sample preparation/digestion protocols reveals tube-gel being a fast and repeatable method for quantitative proteomics. Proteomics, 16(23), 2953-2961. doi: 10.1002/pmic.201600288

[28] MacLean, B., Tomazela, D. M., Shulman, N., Chambers, M., Finney, G. L., Frewen, B., ... MacCoss, M. J. (2010). Skyline: an open source document editor for creating and analyzing targeted proteomics experiments. Bioinformatics, 26(7), 966-968. doi: 10.1093/bioinformatics/btq054

[29] Reiter, L., Rinner, O., Picotti, P., Huttenhain, R., Beck, M., Brusniak, M. Y., . . Aebersold, R. (2011). mProphet: automated data processing and statistical validation for large-scale SRM experiments. Nat Methods, 8(5), 430-435. doi: 10.1038/nmeth.1584

[30] Ramsay, J. O. (1988). Monotone Regression Splines in Action. Statistical Science, 3(4), 425-441.

[31] Wang, W., \& J., Y. (2018). splines2: Regression Spline Functions and Classes. URL https://CRAN.Rproject.org/package=splines $2 . R$ package version 0.2.8.

[32] Mullen, K. M., \& Van Stokkum, I. H. M. (2012). nnls: The Lawson-Hanson algorithm for nonnegative least squares (NNLS). URL https://CRAN.R-project.org/package=nnls. $R$ package version 1.4.

[33] Sarkar, D. (2008). Lattice: Multivariate Data Visualization with R. New York: Springer.

[34] Team, R. C. (2018). R: A Language and Environment for Statistical Computing. R Foundation for Statistical Computing, Vienna. URL http://www.R-project.org. 
[35] de Mendiburu, F. (2019). agricolae: Statistical Procedures for Agricultural Research. $R$ Package Version 1.3-0. URL https://CRAN.R-project.org/package=agricolae.

[36] Gallien, S., Duriez, E., Demeure, K., \& Domon, B. (2013). Selectivity of LC-MS/MS analysis: implication for proteomics experiments. J Proteomics, 81, 148-158. doi: 10.1016/j.jprot.2012.11.005

[37] Wisniewski, J. R., Ostasiewicz, P., Dus, K., Zielinska, D. F., Gnad, F., \& Mann, M. (2012). Extensive quantitative remodeling of the proteome between normal colon tissue and adenocarcinoma. Mol Syst Biol, 8, 611. doi: 10.1038/msb.2012.44

[38] Wisniewski, J. R., \& Rakus, D. (2014). Multi-enzyme digestion FASP and the 'Total Protein Approach'-based absolute quantification of the Escherichia coli proteome. J Proteomics, 109, 322-331. doi: 10.1016/j.jprot.2014.07.012

[39] Listrat, A., Gagaoua, M., Normand, J., Andueza, D. J., Gruffat, D., Mairesse, G., . . Picard, B. (2020). Are there consistent relationships between major connective tissue components, intramuscular fat content and muscle fibre types in cattle muscle? Animal, 14(6), 1204-1212. doi: $10.1017 / \mathrm{S} 1751731119003422$

[40] Kockmann, T., Trachsel, C., Panse, C., Wahlander, A., Selevsek, N., Grossmann, J., . . Schlapbach, R. (2016). Targeted proteomics coming of age - SRM, PRM and DIA performance evaluated from a core facility perspective. Proteomics, 16(15-16), 2183-2192. doi: 10.1002/pmic.201500502

[41] Faktor, J., Sucha, R., Paralova, V., Liu, Y., \& Bouchal, P. (2017). Comparison of targeted proteomics approaches for detecting and quantifying proteins derived from human cancer tissues. Proteomics, 17(5). doi: 10.1002/pmic.201600323

[42] Perez-Riverol, Y., Csordas, A., Bai, J., Bernal-Llinares, M., Hewapathirana, S., Kundu, D. J., . . . Vizcaino, J. A. (2019). The PRIDE database and related tools and resources in 2019: improving support for quantification data. Nucleic Acids Res, 47(D1), D442-D450. doi: 10.1093/nar/gky1106 
[43] Kaspric, N., Picard, B., Reichstadt, M., Tournayre, J., \& Bonnet, M. (2015). ProteINSIDE to Easily Investigate Proteomics Data from Ruminants: Application to Mine Proteome of Adipose and Muscle Tissues in Bovine Foetuses. PLoS One, 10(5), e0128086. doi: 10.1371/journal.pone.0128086 


\section{Figures legends}

Figure 1: Comparison of the limit of detection, limits of quantification and dynamic range obtained in PRM, SRM and SWATH-MS. Eight-point calibration curves of 20 accurately quantified SIL peptides were established for each assay. (A) Limit of detection (LOD). (B) Lower limit of quantification (LLOQ). (C) Upper limit of quantification (ULOQ). (D) Dynamic range.

Figure 2: Comparison of the accuracy and the linearity performances of PRM, SRM and SWATH-MS. (A-C) Comparison between SRM and PRM (A), SWATH-MS and PRM (B) and SWATH-MS and SRM (C) using the quantification values commonly obtained by the three strategies, while excluding the peptide NNLGELINTLNAAK (TPI1) from the assay $(\mathrm{N}=310)$. The solid black line represents the identity line, and the red dotted line the linear regression obtained for the two compared methods. (D-F) Boxplots of the peptide mol amount per $\mu \mathrm{g}$ of muscular proteins obtained in SRM relative to those obtained in PRM ( $D ; N=339)$, those in SWATH-MS relative to those obtained in PRM $(E ; N=720)$, and those in SWATH-MS relative to those in SRM ( $F ; \mathrm{N}=344)$. The black diamond represents the mean, and the grey dotted line the expected value of 1 .

Figure 3: Comparison of the precision and reproducibility performances of PRM, SRM and SWATH-MS strategies and platforms. (A) Precision. Boxplots of the coefficients of variation (CV) on the light/heavy signal obtained for the targeted peptides in the periodically injected quality control sample in PRM (N $=20)$, SRM $(N=20)$ and SWATH-MS ( $=19)$. (B) Reproducibility. Boxplots of the CV on heavy peptide retention time in the samples' cohort acquired in PRM on a nanoACQUITY UPLC-Q-Exactive Plus platform $(\mathrm{N}=20)$, SRM on a Dionex Ultimate 3000-TSQ Vantage platform $(\mathrm{N}=20)$, and SWATH-MS on a eksigent nanoLC 400-TripleTOF 6600 platform $(\mathrm{N}=19)$. 
Figure 4: Monotone spline smoothing and prediction for log-transformed peptide quantity according to log-transformed peptide intensity. One of the 64 samples is represented here. Red values are the predicted values for the observed data represented in black. All 64 graphs can be found in Supporting Information. 
Table

Table 1: Protein abundances (ng/ $\mathrm{\mu g}$ of proteins) assayed by PRM and SWATH-MS for the 51 samples composed of Longissimus thoracis (LT), Semimembranosus

(SM) and Semitendinosus (ST) muscles. SD: Standard deviation. Values followed by different letters $(\mathrm{a}, \mathrm{b}, \mathrm{c})$ are significantly different from each other at $P \leq$

0.05. Gene ontology annotations within the biological process category were identified using the PROTEINside werb service (https://www.proteinside.org/; $\left.{ }^{[43]}\right)$. 


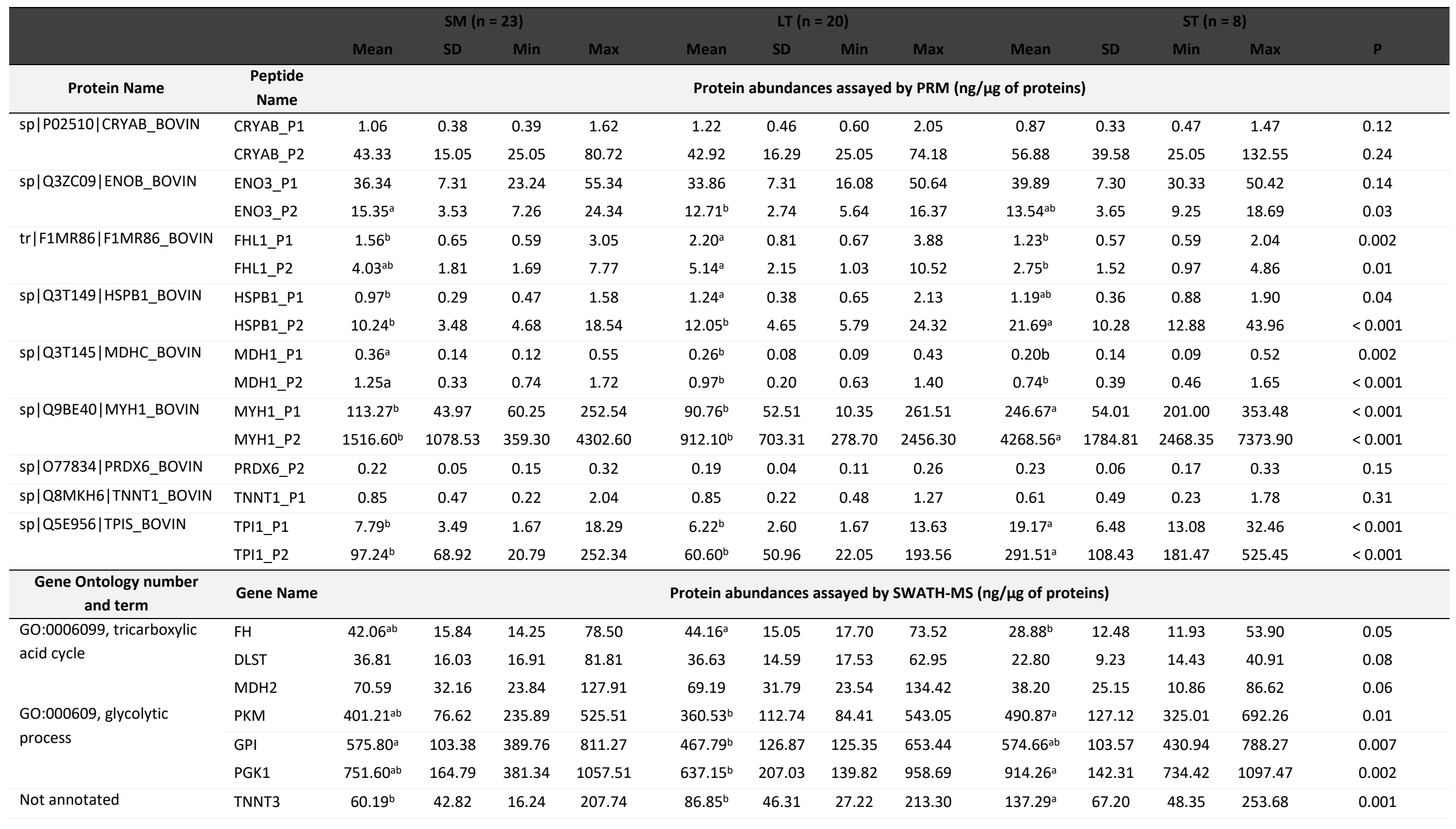




\begin{tabular}{|c|c|c|c|c|c|c|c|c|c|c|c|c|c|c|}
\hline $\begin{array}{l}\text { GO:0006635, fatty acid } \\
\text { beta-oxidation }\end{array}$ & ECHS1 & $47.27^{a b}$ & 19.01 & 16.53 & 84.17 & $59.92^{\mathrm{a}}$ & 35.97 & 22.93 & 179.49 & $32.256^{b}$ & 15.21 & 11.47 & 63.29 & 0.05 \\
\hline $\begin{array}{l}\text { GO:0006122, mitochondrial } \\
\text { electron transport, } \\
\text { ubiquinol to cytochrome }\end{array}$ & UQCRC2 & 32.20 & 11.48 & 16.61 & 59.92 & 37.42 & 14.36 & 16.96 & 64.07 & 23.72 & 9.52 & 10.89 & 35.34 & 0.08 \\
\hline $\begin{array}{l}\text { GO:0006123, mitochondrial } \\
\text { electron transport, } \\
\text { cytochrome c to oxygen }\end{array}$ & $\begin{array}{l}\text { COX5A } \\
\text { MT_CO2 }\end{array}$ & $\begin{array}{l}36.68^{\mathrm{a}} \\
39.22\end{array}$ & $\begin{array}{l}13.25 \\
19.69\end{array}$ & $\begin{array}{l}15.12 \\
7.40\end{array}$ & $\begin{array}{l}60.79 \\
81.95\end{array}$ & $\begin{array}{l}36.01^{\mathrm{ab}} \\
43.65\end{array}$ & $\begin{array}{l}14.02 \\
17.16\end{array}$ & $\begin{array}{l}16.05 \\
17.34\end{array}$ & $\begin{array}{l}63.61 \\
75.40\end{array}$ & $\begin{array}{l}22.20^{\mathrm{b}} \\
25.64\end{array}$ & $\begin{array}{l}14.67 \\
17.52\end{array}$ & $\begin{array}{l}10.87 \\
14.54\end{array}$ & $\begin{array}{l}53.08 \\
60.39\end{array}$ & $\begin{array}{l}0.05 \\
0.14\end{array}$ \\
\hline
\end{tabular}




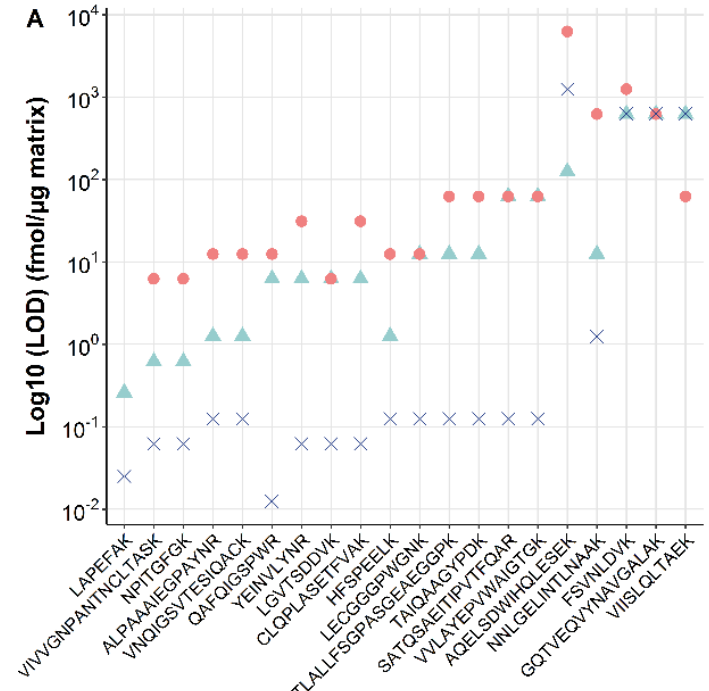

Peptides

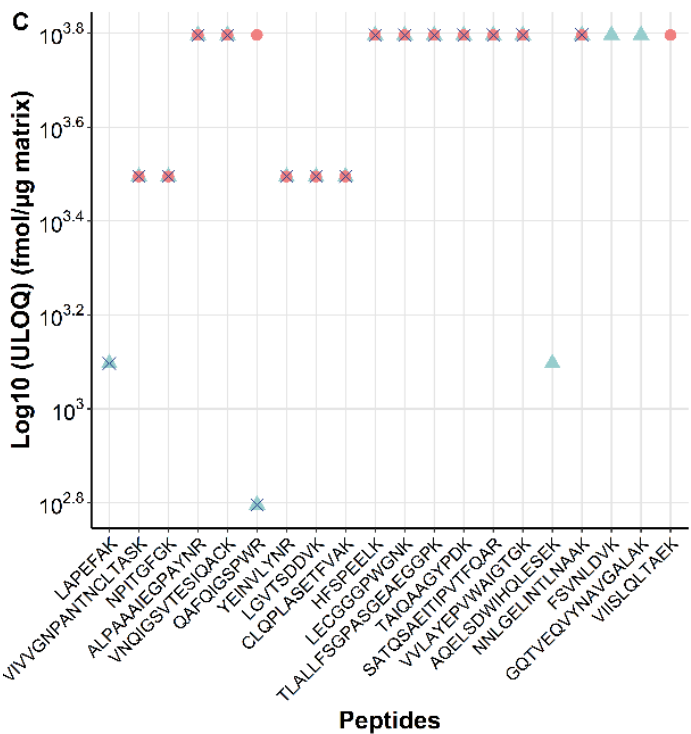

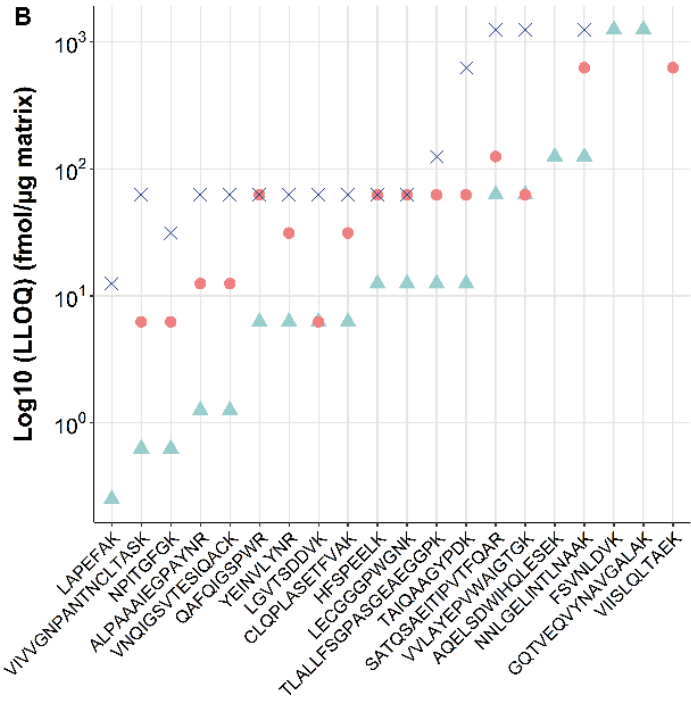

Peptides

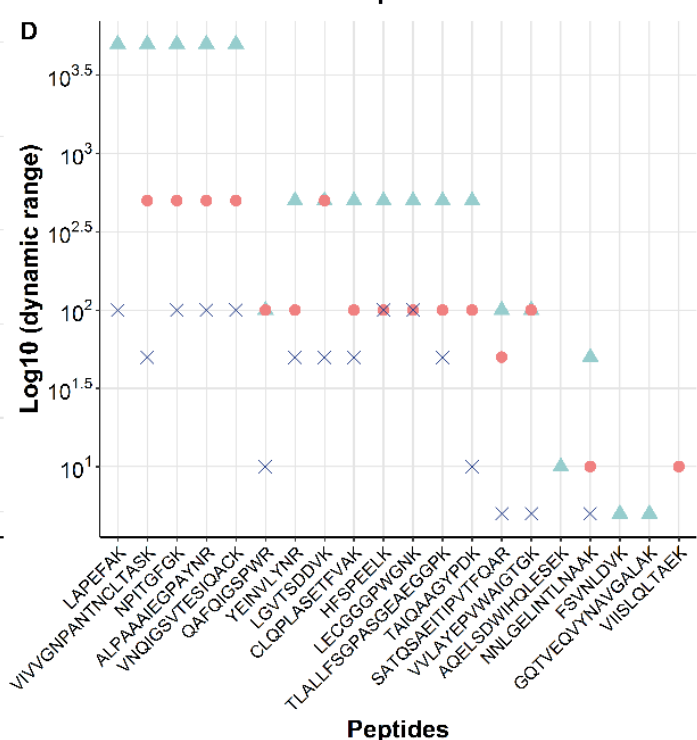

Figure 1
- PRM

SWATH-MS 
A

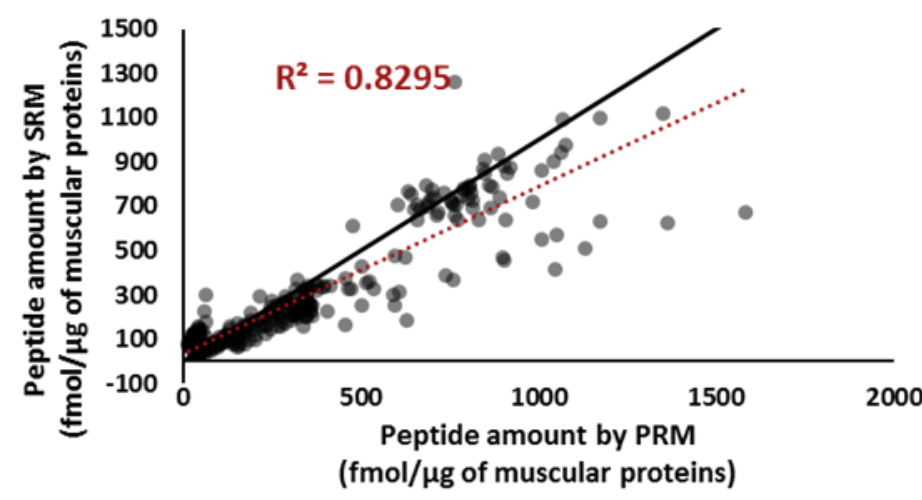

B

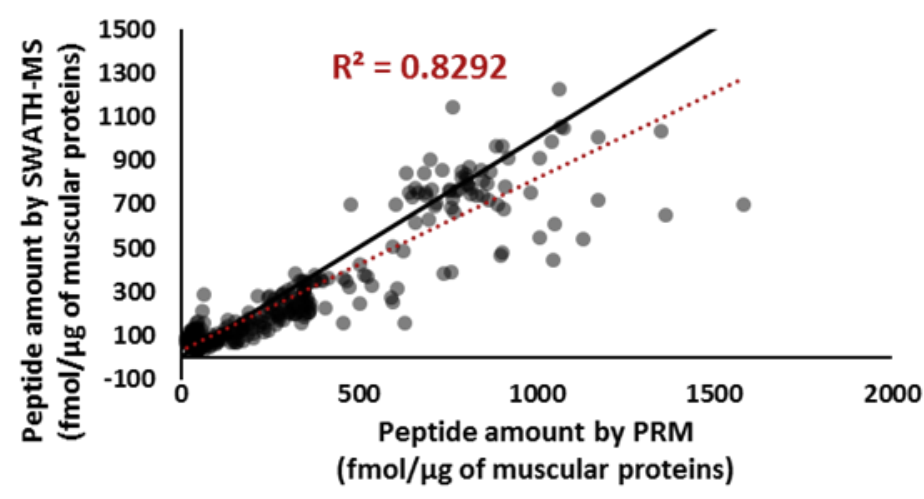

C

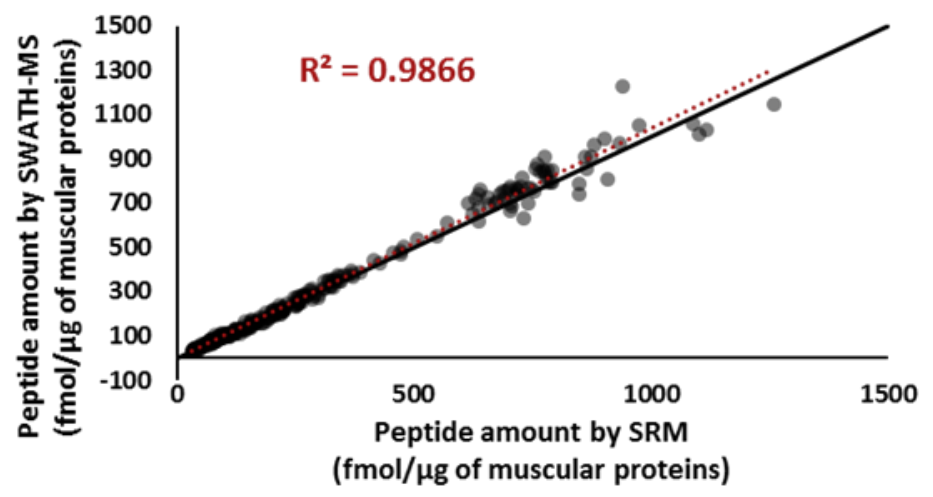

D

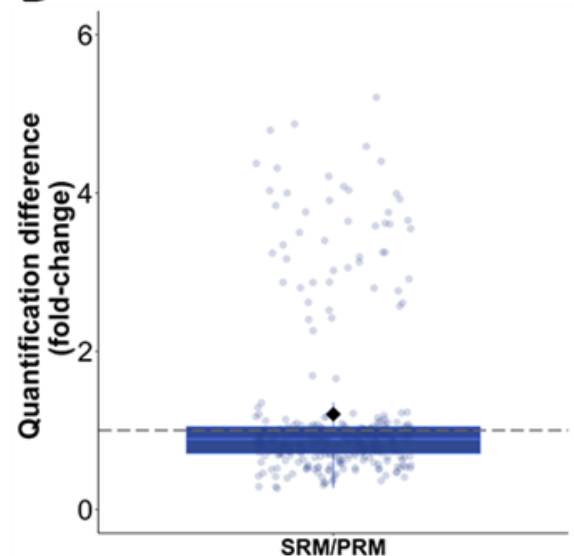

E

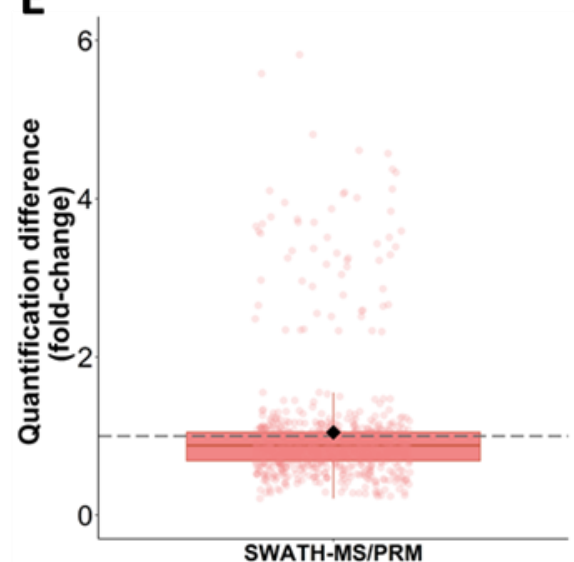

F

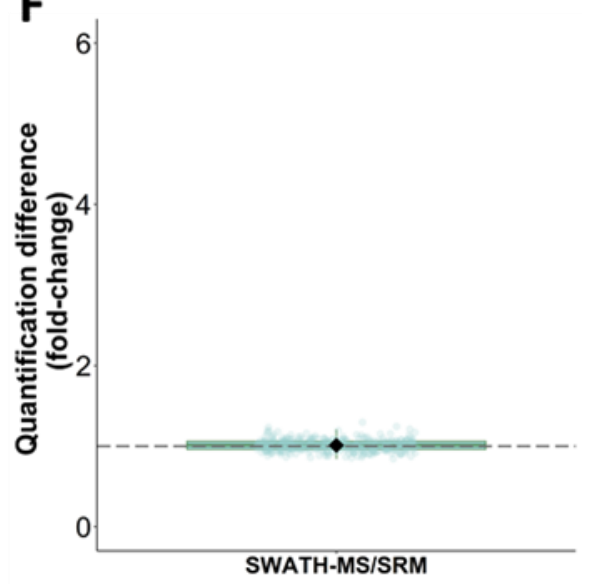

Figure 2 

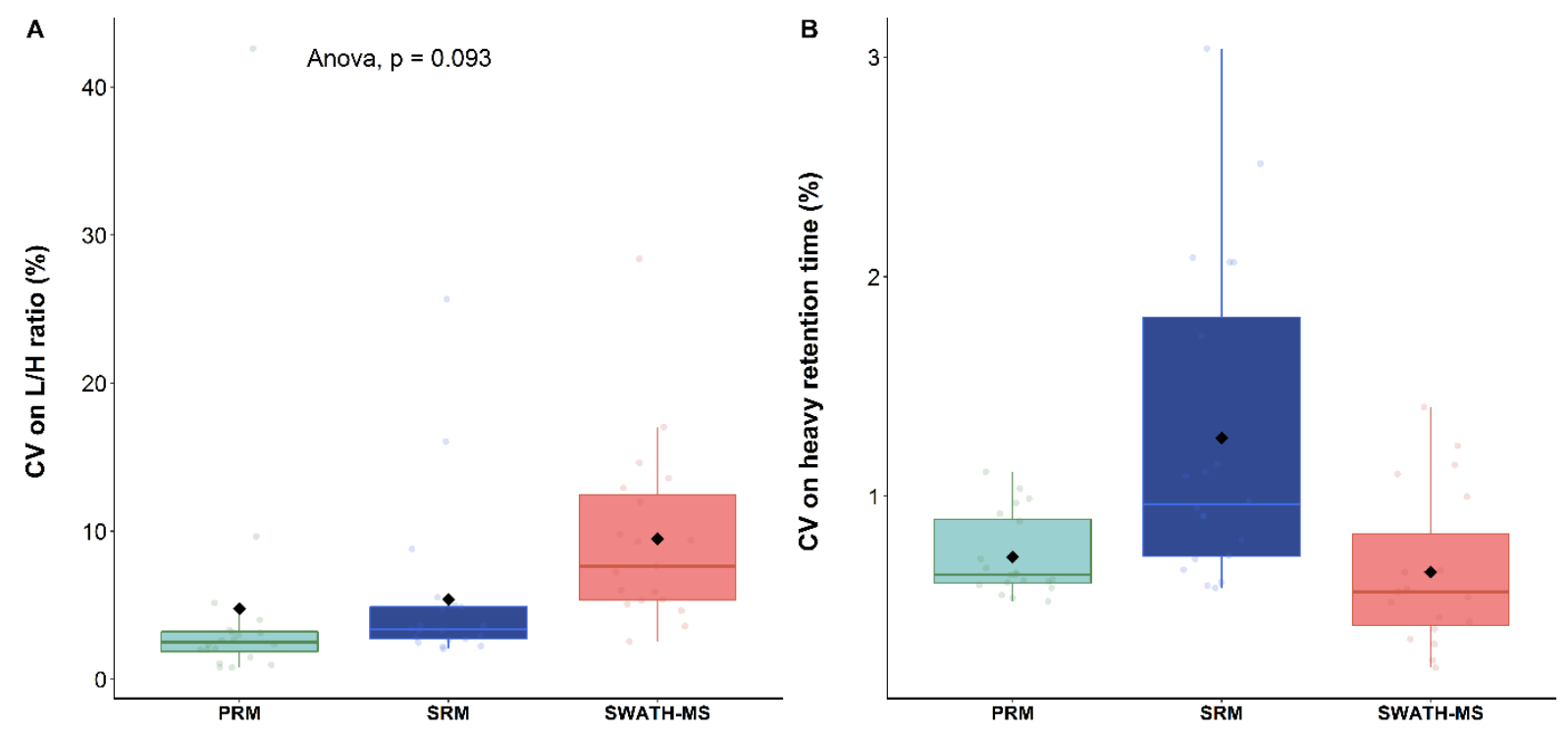

Figure 3 


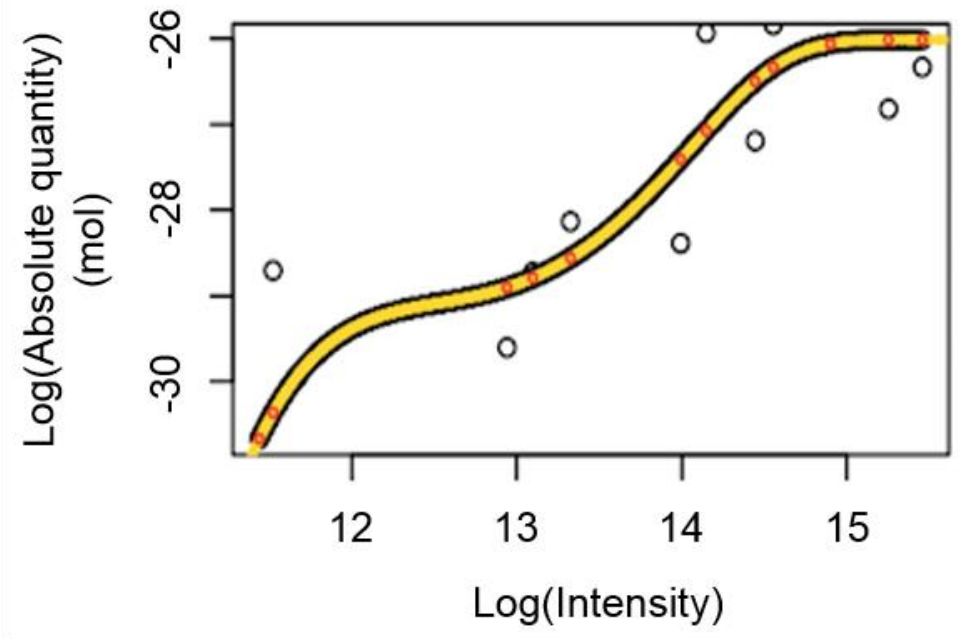

Figure 4 